\title{
Selected Issues Relating to Modeling of Objects and Clothes in the Creating Visualization of Mechanical Hazard during Machine Operation
}

\author{
Dariusz Filipek (i) \\ Central Institute for LabourProtection-NationalResearchInstitute in Warsaw, Czerniakowska 16, 00-701 Warsaw, Poland \\ Correspondence should be addressed to Dariusz Filipek; dafil@ciop.pl
}

Received 10 November 2021; Revised 15 December 2021; Accepted 17 December 2021; Published 25 January 2022

Academic Editor: Marco Roccetti

Copyright (c) 2022 Dariusz Filipek. This is an open access article distributed under the Creative Commons Attribution License, which permits unrestricted use, distribution, and reproduction in any medium, provided the original work is properly cited.

\begin{abstract}
This paper presents selected IT tools of $3 \mathrm{~d}$ s max software functions useful to create visualization of mechanical hazard, especially in object and character modeling aspect. This visualization was elaborated in Central Institute of Labour Protection-National Research Institute. This visualization was elaborated like multimedia OHS training materials from machines operators, OSH employees and employers of mechanical plants, and all institutions of metal machining industry.
\end{abstract}

\section{Introduction}

Film presentation from employees is becoming a more popular way to make more attractive OHS trainings. These films, thanks to their dynamics and memorable message, can be used perfectly by training participants. OHS films can be a supporting or main element of these trainings. Continual changes in the field of law cause the demand from films about this topic.

A good way of making these films is using virtual reality techniques. Especially in the case of serious and fatal accidents, use of visualization methods is the only possible way. Therefore, Central Institute for Labour Protection-National Research Institute elaborated visualizations of mechanical hazard during use of machines for metalworking [1] and woodworking $[2,3]$.

The idea of using virtual reality could be taken from entertainment industry, where computers are often used as a supporting tool in the process of creating film works [4]. Also, the constant progress of virtual reality techniques shows that there are new ways of creating a virtual environment, such as the use of 3D scanners [5]. Progress has also been made in the area of creating virtual characters in recent years [6]. Therefore, as mentioned earlier, there will be a constant demand for films on such topics.
One of the main steps in the process of creating a visualization [7] of mechanical hazard using $3 \mathrm{~d}$ s max software is creation of a virtual environment $[8,9]$. This environment needs presentation of film sequences showing operations following to the accident.

Creation of a virtual environment is an enormously complex and time-consuming process [10]. Modeling $[11,12]$ shapes of objects can be made in different ways. This process begins with a simple object known as "Primitive." Then, the $3 \mathrm{D}$ object is modified to edit mesh level and apply the appropriate modifier. However, some of 3D objects do not model like meshes and polygons only like compound objects. They are created as a result of relevant operations, for example, Boolean operations.

\section{Modeling of Compound 3D Objects}

Compound objects are created based on other previously created objects (at least two). To the most important of compound objects belong Booleans (Figure 1) pro-Boolean, Loft, Connect, and Procutter. Usually, the compound objects used are Boolean objects. They are important elements to improve creating a virtual environment. They are made on mesh objects called "operands." Boolean objects are used (in most of cases) for regular and closed 3D objects about 


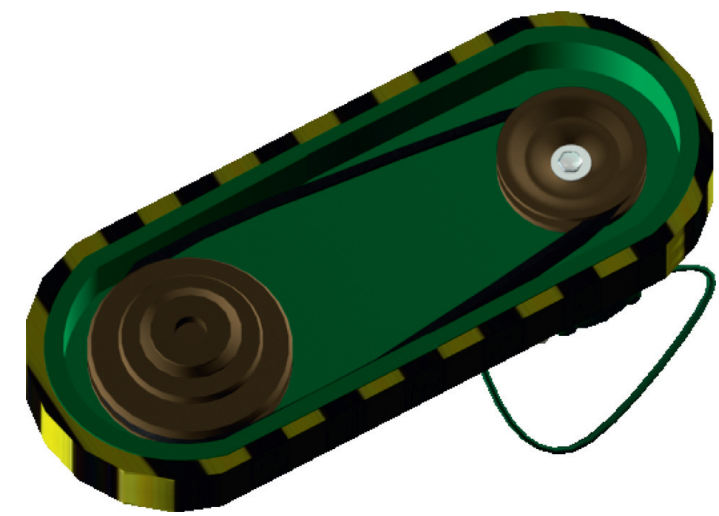

Figure 1: Use of a compound object Boolean from modeling of pulley safety guard of Drill Press.

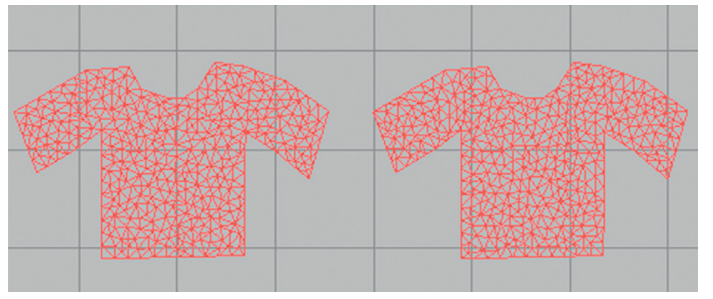

(a)

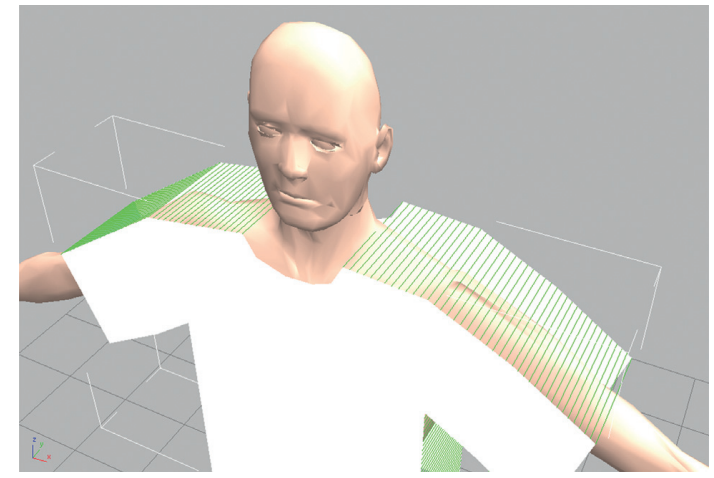

(b)

FIGURE 2: Elements of modifier Garment Maker help modeling of fabric. (a) Fabric mesh created using Dalaunaya algorithm; (b) connection of fabric edges by virtual seams.

defined internal and external surfaces. Boolean operations depend to a large of extent on the axis of $3 \mathrm{D}$ object surface. It means all object surfaces must be directed on outside of the object (does not be inverted normals).

For Boolean operations, at least two objects are needed. The result of this operation will be a new object. Boolean operations have one flaw impact on optimalization of scene. The mesh created as a result of Boolean operations is not of the highest quality. The vertices in the created mesh which was on the border of both objects will be doubled and will be not connected vertices.

Sometimes, Boolean operations may fail because the location of meshes does not allow it. We shall then move one object relative to the other. Boolean operations can be applied for many of $3 \mathrm{D}$ objects but simultaneously can be applied only for two-mesh $3 \mathrm{D}$ objects. If more than two meshes are marked, only the active mesh and previously selected mesh will be used like "operands." Boolean operations work too on materials, UV-textures as a result of which we receive object UV-multimap. It is possibly used for many of Boolean operations:

(i) Union: operation based on connection of $3 \mathrm{D}$ objects. The object obtained is the result of addition one object to another object. (ii) Intersection: this operation creates a new $3 \mathrm{D}$ object. The surface of the new $3 \mathrm{D}$ object is the intersection of both original objects.

(iii) Subtraction: the active $3 \mathrm{D}$ object is subtracted from the second $3 \mathrm{D}$ object.

(iv) Cut object: in practice, this operation is the same like that mentioned above.

\section{Elements of the 3D Modeling Technique in Use to Create Character Clothes}

In the case of a worker 3D model, one of the most important elements to modeling is cloth. Just like reality in the virtual world, we can create models of the virtual clothes. However, we should remember that $3 \mathrm{D}$ mesh object to modeling of virtual cloth must have high enough resolution. It will allow for multiple (similar to reality) folding of a virtual fabric strip. Otherwise, when the mesh has very small resolution, small amount of vertices makes unrealistic fabric kinks and waviness.

The virtual clothes can be sewed similar to clothes in the real world. The model of part dress can be a contour of linesorsplines. Inthisway, wecreatethe panels.Everyvertex(corner point) ofthepanel must bedisconnectedby 
function "Break."Otherwise, amodifierapplied to createavirtualfabricmesh(Garment Maker)willmakeroundingon thecornerpointsofthepanel.

This modifier Garment Maker allows for increase of resolution for the mesh of the cloth object. This is implemented by increase of mesh density determined by Dalaunaya algorithm, Figure 2(a). This algorithm makes randomized division of the mesh into triangles (so-called triangulation). It makes the mesh irregular. This fabric made from an irregular mesh is less susceptible to collapse of fabric edges. It is worth mentioning that the virtual fabrics be created based on "splajns" or object "Plane". Objects type "Primitives" should not be used because this caused doubling of the amount of virtual fabric polygons. Properly selected and positioned panels should be connected by virtual seams, Figure 2(b). However, we should note that corresponding to edges, e.g., the sleeves of a shirt should be of more or less identical sizes. 3D object fabric is created by using a modifier "Cloth." This modifier allows for connecting panels of virtual fabric after defining all seams and allows for simulation of virtual fabric. This modifier also allows for add in and setting of gravitation force and another force which can interact with virtual fabric, e.g., wind force.

\section{Conclusions}

Thanks to the development of virtual reality [13], we can currently create tools from OSH trainings. This can be support tools but also main tools. Development of visualization techniques and changes of $\mathrm{OSH}$ regulations are needed to create visualization of these problems.

Materials of this type permit recognition in noninjury manner by employees all hazards from the places they work in. The visualization help of employees, especially with a short internship, helps get acquainted from hazards on their work places. When creating materials of this type, it is convenient to use appropriate systems and tools. These systems and tools help create such materials.

This paper presents selected IT tools, especially in the context of modeling of compound $3 \mathrm{D}$ objects. This tool was very useful during work on the performance of the virtual environment for needs of visualizations of mechanical hazards. This paper also presents elements related to build 3D characters for visualizations like were elaborated in Central Institute of Labour Protection, National Research Institute.

\section{Data Availability}

The data used to support the findings of this study have been deposited in the Advances in Multimedia repository (DOI).

\section{Conflicts of Interest}

The authors declare no conflicts of interest.

\section{References}

[1] K. Błaszkowski, M. Chmielewski, Z. Jaworski, J. Jelonkowski, J. Kozakiewicz, and W. Milewski, Book of Mechanic Workshop Worker, WNT Warsaw, Warszawa, Poland, 1981.
[2] T. Orlicz, Wood Processing by Cuttingtools, Release Warsaw University of Life Sciences, Warsaw, Poland, 1981.

[3] K. Duchnowski, "Machine Processing, Tools and Basic Carpentry Machines, School and Pedagogical Publications, Warsaw, Poland, 1997.

[4] M. Roccetti, G. Marfia, and M. Zanichelli, "The art and craft of making the Tortellino," Computers in Entertainment, vol. 8, no. 4, 2010.

[5] H. M. Qassim and H. Lakany, "Virtual environment modelling using simulated laser scanners," in Proceedings of the 2019 2nd International Conference on Electrical, Communication, Computer, Power and Control Engineering (ICECCPCE), pp. 143-147, Mosul, Iraq, 2019.

[6] K. TchaTokey, O. Christmann, E. Laup-Escande, G. Laup, and S. Richr, "Towards a model of user experience in immersive virtual environments," Advances in Human-Computer Interaction, vol. 2018, Article ID 7827286, 10 pages, 2018.

[7] D. Kalwasiński and D. Filipek, "Multimedia visualizations of mechanical hazards connected with using machinery in the metal industry," Occupational Safety, vol. 11, pp. 14-17, 2011.

[8] K. Myrcha, D. Kalwasiński, and A. Saulewicz, "Modelling of mechanical hazards occurring in store-rooms," in Proceedings of the XI International School of Computer-Aided Design, Manufacturing and Operation, Military University of Technology, Warsaw, Poland, May 2007.

[9] G. Zulch and T. Grieger, "Modelling of occupational health and safety aspects in the digital factory," Computers in Industry, vol. 56, pp. 384-392, 2005.

[10] D. Kalwasiński and K. Myrcha, Virtual Environment for Inter active Simulation of Lathe Operations, Mechanik-Scientific Technical Monthly Magazine, Warsaw, Poland, 2010.

[11] J. Pasek, Modeling and Animation in 3DS Max, Helion, Gliwice, Poland, 2007.

[12] K. L. Murdock, 3ds Max-Modeling Techniques, HELION, Gliwice, Poland, 2002.

[13] Immersive Technologies, Immersive Technologies Releases Next Generation Advanced Equipment Simulators for Both Surface and Underground Mining, http://www.immersivetechnologies. com/news/news2010/news_2010_07.htm, 2010. 